\title{
Primeiro relato de uma criança brasileira portadora da mutação G188E do gene da lipoproteína lipase
}

\author{
First report of a Brazilian child carrying the G188E mutation of lipoprotein lipase gene
}

\author{
Raquel Tiemi Takata ${ }^{1}$, Roberto Schreiber ${ }^{2}$, Elizabeth Prado ${ }^{3}$, Marcelo Mori $^{4}$, Eliana Cotta de Faria ${ }^{5}$
}

\section{RESUMO}

Objetivo: Relatar o caso de uma criança com hipertrigliceridemia grave por mutações do gene da lipoproteína lipase.

Descrição do caso: Menino de três anos que apresentou, com um mês de idade, soro lipêmico. Seu perfil lipídico indicou hipertrigliceridemia grave, com concentrações de triglicerídeos plasmáticos iguais a $25000 \mathrm{mg} / \mathrm{dL}$. Foi detectada a mutação G188E no éxon 5 da lipoproteína lipase em homozigose na criança e em heterozigose nos pais.

Comentários: A deficiência da lipoproteína lípase é uma doença de herança autossômica recessiva e esses pacientes evoluem com hipertrigliceridemia grave.

Palavras-chaves: hipertrigliceridemia; criança; lipase lipoproteica.

\section{ABSTRACT}

Objective: To report the case of a child with serious hypertriglyceridemia due to lipase lipoprotein gene mutation.

Case description: A three-year-old boy presented with lipemic serum at one month of age. His lipid profile revealed serious hypertriglyceridemia with plasma triglycerides levels of $25,000 \mathrm{mg} / \mathrm{dL}$. A mutation G188E in éxon 5 of the lipoprotein lipase gene was detected in homozygosis for him and in heterozygosis for his parents.

Comments: The deficiency of the lipoprotein lipase is a recessive autossomal disease that causes severe hypertriglyceridemia.

Keywords: hypertriglyceridemia; child; lipoprotein lipase.
Instituição: Departamento de Patologia Clínica da Faculdade de Ciências Médicas de Campinas e Departamento de Pediatria da Faculdade de Ciências Médicas da Santa Casa de Misericórdia de São Paulo, SP, Brasil

${ }^{1}$ Mestre pela Faculdade de Ciências Médicas da Universidade Estadual de Campinas (Unicamp); Médica Pediatra, Campinas, SP, Brasil

2 Doutor em Biologia Molecular pela Unicamp; Biologista no Núcleo de Medicina e Cirurgia Experimental da Faculdade de Ciências Médicas da Unicamp, Campinas, SP, Brasil.

${ }^{3}$ Professora Doutora do Departamento de Pediatria e Puericultura da Santa Casa de Misericórdia de São Paulo, São Paulo, SP, Brasil

${ }^{4}$ Mestre em Pediatria pela Faculdade de Ciências Médicas da Santa Casa de Misericórdia de São Paulo; Médico Assistente da Gastropediatria do Departamento de Pediatria e Puericultura da Santa Casa de Misericórdia de São Paulo, São Paulo, SP, Brasil

${ }^{5}$ Professora Associada do Departamento de Patologia Clínica da Faculdade de Ciências Médicas da Unicamp, São Paulo, SP, Brasil
Endereço para correspondência:

Raquel Tiemi Takata

Rua Tessália Vieira de Camargo, 126 - Barão Geraldo

CEP 13084-971 - Campinas/SP

E-mail: ratiemi97@yahoo.com.br

Fontes financiadoras: Fundação de Amparo à Pesquisa do Estado de São Paulo (Fapesp), processo n. ${ }^{\circ}$ 2006/03212-5; Conselho Nacional de Desenvolvimento Científico e Tecnológico (CNPq), processo n. ${ }^{\circ}$ 473165/2006-6. Conflitos de interesse: nada a declarar

Recebido em: 19/8/2009

Aprovado em: 26/1/2010 


\section{Introdução}

A incidência da deficiência da lipoproteína lipase (LPL; OMIM 238600) na população é de um em cada 1.000.000 indivíduos ${ }^{(1)}$. Trata-se de doença de herança autossômica recessiva caracterizada por hipertrigliceridemia grave $^{(2)}$. O gene da LPL está localizado no braço curto do cromossomo 8 , na posição 22.

A suspeita diagnóstica pode ser feita durante a coleta de sangue, pela observação do aspecto rosado do sangue do paciente e pelo soro extremamente lipêmico. A principal manifestação clínica é a dor abdominal, que pode ser evidente desde o nascimento ${ }^{(2)}$. $\mathrm{O}$ tratamento consiste na restrição dietética de gordura e carboidratos ${ }^{(1-3)}$.

Uma revisão dos casos da deficiência familiar da LPL mostrou que, das 221 mutações descritas, a mutação G188E foi encontrada em $23,5 \%$ dos casos e $74,6 \%$ das mutações estão localizadas no éxons 5 e 6 . Das mutações detectadas ${ }^{(4)}$, aproximadamente $70 \%$ são de sentido trocado (missense); $10 \%$ são sem sentido (nonsense); $18 \%$ são rearranjos gênicos e $2 \%$ de tipo desconhecido ${ }^{(5)}$.

Este estudo trata da descrição de um caso clínico de uma criança com hipertrigliceridemia grave por deficiência familiar de LPL.

\section{Descrição de caso}

Criança de três anos, sexo masculino, filho único de pais consanguíneos, sem antecedentes na família de doenças similares. A criança é produto de primeira gestação sem intercorrências. Nasceu de parto vaginal, em boas condições. O peso ao nascer foi de $3800 \mathrm{~g}$, a estatura $49 \mathrm{~cm}$ e o perímetro cefálico $37 \mathrm{~cm}$, correspondendo ao percentil 50 de cada variável para idade e sexo.

O paciente, natural e procedente de São Paulo, foi levado pela mãe ao Pronto-Socorro Infantil da Santa Casa de Misericórdia de São Paulo aos 25 dias de vida por apresentar febre $\left(38\right.$ a $\left.39^{\circ} \mathrm{C}\right)$ e tosse durante uma semana. Na história clínica, não havia queixas de vômitos, diarreia, nem distensão abdominal. A criança recebia aleitamento materno exclusivo e a mãe relatava diminuição da ingesta às mamadas.

Ao exame físico, apresentava-se em regular estado geral, corado, eupneico, hidratado e anictérico. A fontanela anterior era normotensa e media $2 \times 2 \mathrm{~cm}$. A ausculta cardíaca era normal e a pulmonar apresentava estertores subcrepitantes. $\mathrm{O}$ abdome era normotenso, sem distensão, os ruídos hidroaéreos estavam presentes, o fígado se encontrava a $3 \mathrm{~cm}$ do rebordo costal direito e o baço a $2 \mathrm{~cm}$ do rebordo costal esquerdo.

Tabela 1 - Características antropométricas, clínicas e laboratoriais do paciente aos 25 dias e aos 3 anos e de sua família na avaliação inicial

\begin{tabular}{lcccc}
\hline & \multicolumn{2}{c}{ Paciente } & Pai & Mãe \\
\hline Idade & 25 dias & 3 anos & 23 anos & 23 anos \\
IMC $\left(\mathrm{kg} / \mathrm{m}^{2}\right)$ & 15 & 15 & 21 & 23 \\
TG $(\mathrm{mg} / \mathrm{dL})$ & 25000 & 922 & 71 & 107 \\
TC $(\mathrm{mg} / \mathrm{dL})$ & nd & 194 & 161 & 189 \\
LDL-C $(\mathrm{mg} / \mathrm{dL})$ & nd & 24 & 107 & 131 \\
HDL-C $(\mathrm{mg} / \mathrm{dL})$ & nd & 20 & 40 & 37 \\
VLDL-C $(\mathrm{mg} / \mathrm{dL})$ & nd & 47 & 14 & 21 \\
Lp $(a)(\mathrm{mg} / \mathrm{dL})$ & nd & 2,36 & 2,36 & 2,36 \\
Glicose $(\mathrm{mg} / \mathrm{dL})$ & nd & 98 & 81 & 78 \\
Insulina $(\mu / \mathrm{l} / \mathrm{mL})$ & nd & 3,5 & 2 & 2 \\
IL-6 $(\mathrm{pg} / \mathrm{mL})$ & nd & 0,01 & 0,01 & 0,01 \\
PCR $(\mathrm{mg} / \mathrm{dL})$ & nd & 3,1 & nd & nd \\
\hline
\end{tabular}

IMC: índice de massa corpórea; TG: triglicerídeos; TC: colesterol total; LDL-C: LDL-colesterol; HDL-C: HDL-colesterol; VLDL-C: VLDL-colesterol; Lp(a): lipoproteína (a); PCR: proteína C-reativa; IL-6: interleucina 6; nd: não determinado.

Valores de referência segundo III Diretrizes Brasileiras sobre Dislipidemias e Prevenção da Aterosclerose: C<170mg/dL, LDL (<110mg/dL), HDLC $>45 \mathrm{mg} / \mathrm{dL}$ ( $<10$ anos), $\mathrm{HDL}>35 \mathrm{mg} / \mathrm{dL}$ ( $>10$ anos) e TG<100mg/dL ( $<10$ anos) e TG<130 mg/dL(10-19 anos); IL-6 $<0,01 \mathrm{mg} / \mathrm{dL}$ e $P C R<2,1 \mathrm{mg} / \mathrm{dL}$. 
Para complementar o diagnóstico, foram solicitados exames de imagens. $\mathrm{O}$ raio $\mathrm{X}$ de tórax revelou broncopneumonia; o raio $\mathrm{X}$ de abdome mostrou aumento do fígado; e a ultrassonografia de abdome revelou hepatomegalia. $\mathrm{Na}$ investigação, foram ainda solicitados exames laboratoriais, notando-se soro lipêmico. O perfil lipídico do paciente aos 25 dias e depois, aos 3 anos, e o dos pais são mostrados na Tabela 1. Os pais não apresentaram alterações do perfil lipídico.

Após o diagnóstico de hipertrigliceridemia grave, foi iniciada uma dieta com leite desnatado. Como o paciente não seguiu a dieta restritiva de gordura, ele continuou a apresentar hipertrigliceridemia grave. Aos três anos, a criança apresentava crescimento normal (peso e estatura dentro do percentil 50). A estatura nessa idade era de $92 \mathrm{~cm}$, o peso $13 \mathrm{~kg}$ e o perímetro cefálico de $48 \mathrm{~cm}$.

\section{Análise molecular}

O DNA foi extraído de leucócitos periféricos de acordo com Salazar et al $l^{(6)}$. Para identificar a mutação G188E, utilizou-se a amplificação do éxon 5 pela técnica de reação em cadeia da polimerase (PCR), conforme descrito por Monsalve $e t{ } l^{(7)}$ e tratada com a enzima de restrição AVA-II (Invitrogen, USA).

No heredograma (Figuras 1A e 1B), indicam-se os pais e o paciente: a homozigose no paciente e heterozigose nos pais (bandas 2 e 4 na eletroforese, respectivamente do pai e da mãe). A mutação pode ser designada como G188E ou, indicando a troca do aminoácido glicina $(G)$ por ácido glutâmico (E), como GLY188GLU.

\section{Comentários}

A deficiência familiar da lipoproteína lipase é uma doença herdada de modo autossômico recessivo ${ }^{(8)}$. Sua incidência na população geral é de cerca de 1:1.000.000 indivíduos ${ }^{(4)}$. $\mathrm{O}$ gene da LPL apresenta peso molecular de $35 \mathrm{~Kb}$, contém dez éxons e é mapeado no cromossomo $8 \mathrm{p} 22^{(9)}$. A lipoproteína lipase é a enzima-chave na hidrólise dos triglicerídeos e indivíduos com a deficiência dessa enzima evoluem com hipertrigliceridemia grave ${ }^{(10,11)}$. Quanto ao quadro clínico, os pacientes podem apresentar, desde a infância, sintomas como dor abdominal, xantomas eruptivos e pancreatite ${ }^{(11)}$. O diagnóstico da deficiência da lipoproteína lipase pode ser realizado pela ausência da atividade da lipoproteína lipase pós-heparina. A atividade da lipoproteína lipase é determinada 15 minutos após a injeção endovenosa de 100UI/kg de heparina.O tratamento consiste na restrição da ingestão de gorduras, ou seja, preconiza-se a ingestão inferior a $20 \mathrm{~g} / \mathrm{dia}^{(12,13)}$.

A mutação em homozigose G188E do gene da lipoproteína lipase em um menino brasileiro de três anos que apresentou soro lipêmico com um mês de idade foi relatada neste trabalho. Seu perfil lipídico revelou hipertrigliceridemia grave, com concentração plasmática de triglicerídeos de $25.000 \mathrm{mg} / \mathrm{dL}$. A mesma mutação foi detectada em heterozigose em seus pais. O paciente apresentou-se de maneira similar à descrita na literatura, em que homozigotos para a mutação G188E do gene da lipoproteína lipase evidenciam hipertrigliceridemia no momento do diagnóstico. Após o diagnóstico de hipertrigliceridemia grave, iniciou-se uma dieta com leite desnatado. Como o paciente não seguiu a dieta restritiva de gordura, continuou a apresentar hipertrigliceridemia grave e, no seguimento, mostrou crescimento normal (peso e estatura no percentil 50).

A extrema raridade da doença faz com que a detecção de consanguinidade entre os pais seja elevada. Tal fato ocorreu na presente família, na qual os pais eram primos em primeiro grau. O aconselhamento genético deve ser realizado devido à alta probabilidade de recorrência (25\%). Também é indicada a detecção de heterozigotos entre os consanguíneos dos pais da criança.

É importante acompanhar os pacientes com hipertrigliceridemia grave do ponto de vista clínico, laboratorial e radiológico, pelo risco de evoluírem com pancreatite aguda e/ou com doença aterosclerótica, para a qual tem sido considerada fator de risco independente ${ }^{(14-16)}$. Dentre

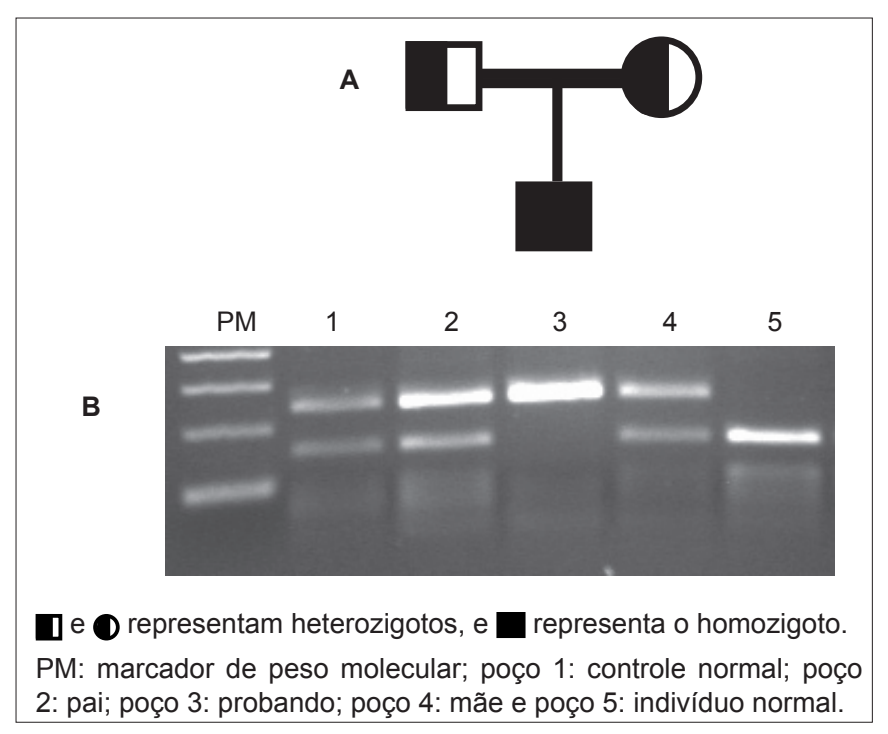

Figura 1 - (A.) Heredograma. (B.) Detecção da mutação G188E do probando e seus pais por eletroforese em gel de agarose. 
as hipertrigliceridemias primárias, aquelas com deficiência da lipoproteína lipase e mutação no códon 188 apresentam risco de problemas coronarianos cinco vezes maior, em comparação às outras mutações associadas ao gene da $\operatorname{LPL}^{(17,18)}$. Os mecanismos que poderiam explicar a aterogenicidade da hipertrigliceridemia são: aumento sérico de quilomícrons remanescentes, elevação de remanescentes de VLDL (very low density lipoprotein) e de lipoproteínas de baixa densidade pequenas e densas, com deposição destas no espaço subendotelial, diminuição de HDL-colesterol (HDL-C) e alterações nos fatores da coagulação (aumento de PAI-1 e diminuição da fibrinólise $)^{(19,20)}$.

Os pacientes com hipertrigliceridemia familiar apresentam produção hepática aumentada de triglicerídeos (níveis em torno de 500 a $600 \mathrm{mg} / \mathrm{dL}$ ) e podem ter redução relativa da atividade de LPL. Eles também desenvolvem diminuição de HDL-C e maior risco da doença arterial coronariana ${ }^{(17,19)}$. Fatores agravantes, como dieta calórica, obesidade, ingestão alcoólica, diabetes melito e hiperuricemia aumentam o risco desses pacientes desenvolverem hipertrigliceridemia ${ }^{(21)}$.

\section{Referências bibliográficas}

1. Mc Donnel MG, Young IS, Nicholls DP, Archbold GP, Graham CA. Genetics of the lipoprotein lipase gene and hypertriglyceridaemia. $\mathrm{Br} \mathrm{J}$ Biomed $\mathrm{Sci}$ 2003;60:84-8.

2. Sugandhan $\mathrm{S}$, Khandpur S, Sharma VK. Familial chylomicronemia syndrome. Pediatr Dermatol 2007;24:323-5.

3. Mohandas MK, Jemila J, Ajith Krishnan AS, George TT. Familial chylomicronemia syndrome. Indian J Pediatr 2005;72:181.

4. Reina M, Brunzell JD, Deeb SS. Molecular basis of familial chylomicronemia: mutations in the lipoprotein lipase and apoliprotein C-II genes. J Lipid Res 1992;33:1823-32.

5. Gilbert B, Rouis M, Griglio S, de Lumley L, Lauplaud P. Lipoprotein lipase (LPL) deficiency: a new patient homozygote for the preponderant mutation Gly188Glu in the human LPL gene and review of reported mutations: $75 \%$ are clustered in exon 5 and 6. Ann Genet 2001;44:25-32.

6. Salazar LA, Hirata MH, Cavalli SA, Machado MO, Hirata RD. Optimized procedure for DNA isolation from fresh and cryopreserved clotted human blood useful in clinical molecular testing. Clin Chem 1998;44:1748-50.

7. Monsalve MV, Henderson H, Roederer G, Julien P, Deeb S, Kastelein JJ et al. A missense mutation at codon 188 of the human lipoprotein lipase gene is a frequent cause of lipoprotein lipase deficiency in persons of different ancestries. J Clin Invest 1990;86:728-34.

8. Rouis M, Dugi KA, Previato L, Patterson AP, Brunzel JD, Brewer BH et al. Therapeutic response to medium-chain triglycerides and -3 fatty acids in a patient with the familial chylomicronemia syndrome. Arterioscler Thromb Vasc Biol 1997:17:1400-6.

9. Merkel M, Eckel RH,Goldberg IJ. Lipoprotein lipase: genetics, lipid uptake, and regulation. J Lipid Res 2002;43:1997-2006.

10. Kawashiri M, Higashikata T, Mizuno M, Takata M, Katsuda S, Mina K et al. Long-term course of lipoprotein lipase (LPL) deficiency due to homozygous $\mathrm{LPL}$ (Arita) in a patient with recurrent pancreatitis, retained glucose tolerance, and atherosclerosis. J Clin Endocrinol Metab 2005;90:6541-4.
O uso de marcadores da inflamação na aterosclerose pode auxiliar o diagnóstico. A criança descrita apresentou aumento plasmático de proteína C-reativa (PCR) e da interleucina 6 (IL-6), sugerindo já existir repercussão pró-aterogênica. Não foi possível realizar a medida da atividade da LPL pósheparina nessa criança, pois os pais não autorizaram.

O relato deste caso enfatiza a importância do diagnóstico e do tratamento precoce da hipertrigliceridemia para prevenir, ainda na infância, o início da aterosclerose e da pancreatite aguda.

\section{Agradecimentos}

Ao laboratório de Bioquímica Clínica do Hospital de Clínicas e ao Laboratório de Lípides da Faculdade de Ciências Médicas da Unicamp; em especial, a Mirian Danelon, pelo excelente apoio técnico. Também ao suporte do Conselho Nacional de Desenvolvimento Científico e Tecnológico (CNPq) e da Fundação de Amparo à Pesquisa do Estado de São Paulo (Fapesp).
11. Nordestgaard BG, Abildgaard S, Wittrup HH, Steffensen R, Jensen G, TybjaergHansen A. Heterozygous lipoprotein lipase deficiency: frequency in the general population, effect on plasma lipid levels, and risk of ischemic heart disease. Circulation 1997;96:1737-44.

12. Peterson J, Ayyobi AF, Ma Y, Henderson H, Reina M, Deeb SS et al. Structural and functional consequences of missense mutations in exon 5 of the lipoprotein lipase gene. J Lipid Res 2002;43:398-406.

13. Hodge D, Stringer MD, Puntis JW. Lipoprotein lipase deficiency: benefits and limitations of a novel therapeutic surgical approach. J Pediatr Gastroenterol Nutr 2001:32:593-5.

14. Wittrup HH, Tybjaerg-Hansen A, Noordestgaard BG. Lipoprotein lipase mutations, plasma lipids and lipoproteins and risk of ischemic heart disease. A meta-analysis. Circulation 1999;99:2901-7.

15. Breslow JL. Genetics of lipoprotein abnormalities associated with coronary artery disease susceptibility. Annu Rev Genet 2000;34:233-54.

16. Ravi GR, Pradeepa R, Mohan V. Hypertriglyceridemia and coronary artery disease--an update. Indian Heart J 2004;56:21-6.

17. Nordestgaard BG, Abildgaard S, Wittrup HH, Steffensen R, Jensen G, TybjærgHansen A. Heterozygous lipoprotein lipase deficiency: frequency in the general population, effect on plasma lipid levels, and risk of ischemic heart disease. Circulation. 1997;96:1737-1744.

18. Evans V, Kastelein JJ. Lipoprotein lipase deficiency - rare or common? Cardiovasc Drugs Ther 2002;16:283-7.

19. Freitas EV. Triglícerídeos e doença arterial coronariana. Rev SOCERJ 2004;17:45-9.

20. Fischer R, Humphries SE, Talmud PJ. Common variation in the lipoprotein lipase gene: effects on plasma lipids and risk of atherosclerosis. Atherosclerosis 1997;135:145-59.

21. Kane JP, Malloy MJ. Distúrbios do metabolismo das lipoproteínas. In: Greenspan FS, Strewler GJ, editores. Endocrinologia básica e clínica. Rio de Janeiro: Guanabara Koogan; 2000. p. 501-21. 